\title{
Seleção de famílias de feijoeiro resistente à antracnose e à mancha-angular
}

\author{
Marcelo Geraldo de Morais Silva(1), João Bosco dos Santos ${ }^{(1)}$ e Ângela de Fátima Barbosa Abreu(1)
}

(1)Universidade Federal de Lavras, Dep. de Biologia, Caixa Postal 3037, CEP 37200-000, Lavras, MG. E-mail: mcoro2003@yahoo.com.br, jbsantos@ufla.br, afbabreu@ufla.br

\begin{abstract}
Resumo - O objetivo deste trabalho foi identificar famílias de feijoeiro com resistência a Colletotrichum lindemuthianum e Phaeoisariopsis griseola e com outros fenótipos agronômicos desejáveis. As famílias utilizadas foram obtidas do cruzamento entre a linhagem H91, portadora de três alelos de resistência à antracnose, com três famílias $\mathrm{F}_{2: 5}$ resistentes à mancha-angular, derivadas da cultivar Jalo EEP 558. Foi utilizado o delineamento látice quadrado em todos os experimentos. Inicialmente, foram avaliadas 144 famílias $\mathrm{F}_{2: 3}$, no inverno de 2004, em Lavras, MG, com base no tipo de grão. Foram selecionadas 80 famílias $\mathrm{F}_{2: 4}$ e avaliadas com a testemunha BRSMG Talismã, no período das águas de 2004/2005, no mesmo local. Considerando-se o tipo de grão e a resistência à mancha-angular e antracnose, foram mantidas 48 famílias $\mathrm{F}_{2: 5}$, que foram avaliadas na seca de 2005, em Lavras e Lambari, MG. Essas 48 famílias passaram por inoculação das raças 2047, 73 e 1545 de C. lindemuthianum, para verificação da presença dos alelos de resistência $\mathrm{Co}_{-} 4^{2}, \mathrm{Co}-5$ e $\mathrm{Co}-7$, respectivamente. Foram identificados genótipos da maioria das 48 famílias, quanto à reação à antracnose, das quais se destacaram quatro, em relação ao tipo de grão semelhante ao 'Carioca', de porte ereto, produtividade elevada e resistência à mancha-angular.
\end{abstract}

Termos para indexação: Colletotrichum lindemuthianum, Phaeoisariopsis griseola, resistência, seleção, caracteres agronômicos.

\section{Selection of common bean families resistant to anthracnose and angular leaf spot}

\begin{abstract}
The objective of this work was to select common bean families resistant to Colletotrichum lindemuthianum and Phaeoisariopsis griseola and, also, with superior agronomical traits. Families used were obtained from crosses of $\mathrm{H} 91$ lineage, bearer of three alleles resistant to anthracnose, and $\mathrm{F}_{2: 5}$ families derived from the cultivar Jalo EEP 558, which is resistant to angular leaf spot. Square lattice design was used in all experiments. Initially the $\mathrm{F}_{2: 3}$ families (144) were evaluated in the winter of 2004, in Lavras county, MG, Brazil, based on grain type. Eighty families $\left(\mathrm{F}_{2: 4}\right)$ were selected and evaluated with the check BRSMG Talismã, in the wet periods of 2004/2005, in the same place. Considering grain type, anthracnose and angular leaf spot resistances, 48 families $\left(\mathrm{F}_{2: 5}\right)$ were selected and evaluated in the dry period of 2005, in two places, Lavras and Lambari, MG. These 48 families were also inoculated with the 2047, 73 and 1545 races of $C$. lindemuthianum, in order to verify the presence of alleles of resitance $\mathrm{Co}-4^{2}, \mathrm{Co}-5$ and $\mathrm{Co}-7$, respectively. Genotypes with anthracnose resistance were identified in most families, four of which are outstanding for their ideal 'Carioca'-like grain, with upright plant, high grain yield and resistance to angular leaf spot.
\end{abstract}

Index terms: Colletotrichum lindemuthianum, Phaeoisariopsis griseola, resistance, selection, agronomical traits.

\section{Introdução}

O feijão é um alimento de grande importância econômica e social para o Brasil, porém a cultura ainda apresenta baixa produtividade média (Indicadores..., 2005), decorrente de vários problemas, entre eles, a ocorrência de doenças.

Entre as doenças do feijoeiro, destacam-se a antracnose, causada pelo fungo Colletotrichum lindemuthianum, e a mancha-angular, causada pelo fungo Phaeoisariopsis griseola. A antracnose pode ocasionar perdas de até $100 \%$, principalmente quando associada a condições ambientais favoráveis e uso de sementes contaminadas (Peloso, 1992). Em relação à mancha-angular, as perdas podem chegar a $70 \%$, o que depende do maior ou menor grau de suscetibilidade das cultivares, das condições ambientais e da patogenicidade dos isolados (Sartorato, 2005). Essas doenças, além de prejudicarem o 
rendimento da cultura, também depreciam a qualidade do produto final para o consumo e uso como sementes.

O controle integrado das doenças é a estratégia ideal. Nesse sistema integrado, uma alternativa prática e econômica é a utilização de cultivares resistentes aos dois patógenos. No entanto, a criação de cultivares resistentes a essas doenças é dificultada pela enorme capacidade de variação patogênica desses fungos, o que resulta no aparecimento de grande número de raças fisiológicas (Rava et al., 1994).

Um dos entraves para o uso da resistência vertical contra os dois patógenos é a existência de grande número de raças, reduzindo a vida útil de uma cultivar com apenas um alelo de resistência. Uma alternativa eficiente para obter uma cultivar com resistência mais duradoura é a piramidação de alelos de resistência, ou seja, transferir para uma única linhagem vários alelos presentes em outras fontes (Kelly \& Miklas, 1998). Na identificação das plantas portadoras de pirâmide, uma dificuldade que se apresenta é a não-disposição de raças do patógeno que as identifique. No entanto, isto pode ser contornado utilizando-se as raças disponíveis e marcadores moleculares (Young et al., 1998; Pereira et al., 2004; Couto, 2005).

Na obtenção de cultivares com pirâmide de alelos de resistência para as duas doenças em questão, outros atributos agronômicos também devem ser considerados. Entre eles, o tipo de grão aceitável pelo consumidor, como o tipo 'Carioca'; porte ereto, que evita perdas na colheita e favorece a colheita mecanizada; e alta produtividade, essencial no processo da obtenção de uma nova cultivar (Pereira et al., 2004).

Este trabalho teve como objetivo identificar famílias de feijoeiro com pirâmide de alelos de resistência a C. lindemuthianum e P. griseola, alta produtividade, porte arbustivo e tipo de grão semelhante ao da cultivar Carioca.

\section{Material e Métodos}

Um dos genitores utilizados foi a linhagem H91, resistente à antracnose (Pereira et al., 2004). Essa linhagem, além de portadora dos alelos $\mathrm{Co}-4^{2}, \mathrm{Co}-5$ e $\mathrm{Co}-7$, é possuidora de caracteres desejáveis tais como porte ereto, grão tipo 'Carioca' e boa produtividade de grãos, porém é suscetível à manchaangular.
Os outros genitores foram três famílias segregantes $\mathrm{F}_{2: 5}$, denominadas $\mathrm{B} 1, \mathrm{~B} 2 \mathrm{e} \mathrm{B} 3$, resistentes à manchaangular derivada da cultivar andina Jalo EEP 558 (Bruzi et al., 2004). O alelo de resistência à manchaangular dessa cultivar tem conferido proteção contra essa doença, no Sul de Minas Gerais, há cerca de três décadas.

Os cruzamentos da linhagem H91 com as três famílias foram realizados em casa de vegetação, no Dep. de Biologia (DBI), da Universidade Federal de Lavras (Ufla). As sementes $F_{2}$ foram semeadas em campo, e cada planta foi colhida individualmente tendo gerado uma família $\mathrm{F}_{2: 3}$.

Os experimentos de campo, para avaliação das famílias, foram conduzidos na área experimental do DBI, no Município de Lavras, e na fazenda experimental da Empresa de Pesquisa Agropecuária de Minas Gerais (Epamig), em Lambari, MG.

$\mathrm{Na}$ safra de inverno de 2004, foram avaliadas 144 famílias $\mathrm{F}_{2: 3}$, no Município de Lavras, MG. $\mathrm{O}$ experimento foi conduzido em delineamento látice quadrado simples $12 \times 12$, com duas repetições e parcelas constituídas de uma linha $1 \mathrm{~m}$ de comprimento.

Foram selecionadas 80 famílias $\mathrm{F}_{2: 4}$, com base no tipo de grão, e avaliadas, novamente, na safra das águas de 2004/2005, em Lavras, com a cultivar BRSMG Talismã como testemunha. O experimento foi conduzido em látice quadrado triplo 9x9, com três repetições e parcelas constituídas de duas linhas de $2 \mathrm{~m}$. As famílias foram avaliadas quanto à produtividade, tipo de grão e resistência à manchaangular.

Das 80 famílias, foram selecionadas 48 , as quais foram avaliadas na safra da seca de 2005, em Lavras e Lambari, com a cultivar BRSMG Talismã. O experimento foi conduzido em látice quadrado triplo $7 \times 7$, com três repetições, e cada parcela foi novamente representada por duas linhas de $2 \mathrm{~m}$.

Todos os experimentos receberam adubação, na semeadura, com $300 \mathrm{~kg} \mathrm{ha}^{-1}$ de $8-28-16\left(\mathrm{~N}-\mathrm{P}_{2} \mathrm{O}_{5}-\mathrm{K}_{2} \mathrm{O}\right)$, mais $150 \mathrm{~kg} \mathrm{ha}^{-1}$ de sulfato de amônio em cobertura, aproximadamente 20 dias depois da emergência. A irrigação por aspersão foi utilizada quando necessária. O espaçamento entre linhas foi de $50 \mathrm{~cm}$ e a densidade de semeadura de 15 sementes por metro linear. 
As avaliações da severidade da mancha-angular foram realizadas com diagrama de notas de 1 (ausência de sintomas visíveis) a 9 (suscetibilidade máxima), proposto por Van Schoonhoven \& Pastor-Corrales (1987). Nas avaliações para tipo de grão, foi utilizada escala descritiva, proposta por Marques-Junior (1997), com notas de 1 (grãos tipo 'Carioca') a 5 (grãos fora do padrão 'Carioca'). As avaliações de porte foram realizadas por meio de um diagrama de notas semelhante ao de Collicchio et al. (1997), com notas de 1 (totalmente ereto) a 5 (prostrado). A produção de grãos foi mensurada em quilogramas por hectare. Os dados de todos os caracteres avaliados tiveram distribuição normal e não foram transformados.

Os dados obtidos das características avaliadas, nos experimentos de campo, foram submetidos a análises de variância individuais e conjuntas. Foram estimadas as herdabilidades no sentido amplo e seus intervalos de confiança, conforme Knapp et al. (1985). Para as análises conjuntas, também foram estimados os ganhos de seleção das famílias superiores. Foram estimadas também as correlações fenotípicas entre os caracteres avaliados.

Para identificar a constituição genética das 48 famílias $\mathrm{F}_{2: 5}$, previamente selecionadas em campo, quanto à presença dos alelos $\mathrm{Co}-4^{2}, \mathrm{Co}-5$ e $\mathrm{Co}-7$ de resistência à antracnose, foram realizadas inoculações de três raças $(2047,1545$ e 73$)$ de C. lindemuthianum.

$\mathrm{O}$ inóculo de cada raça foi preparado de cultura monospórica na concentração de $1,2 \times 10^{6}$ esporos $\mathrm{mL}^{-1}$. Sementes das famílias, dos genitores e das testemunhas resistente (G2333) e suscetível ('Carioca') foram semeadas em bandejas de isopor, com substrato plantimax. Doze plantas de cada família foram utilizadas, e por volta de dez dias após a semeadura, procedeu-se à inoculação, pulverizandose a suspensão de esporos nas faces abaxial e adaxial das folhas primárias com um borrifador. Em seguida, as plântulas foram mantidas em câmaras especiais de inoculação, sob condições controladas de temperatura, luz e umidade, por cinco dias. Depois desse período, as bandejas foram transferidas para um telado, com irrigação intermitente, onde permaneceram até o momento da avaliação dos sintomas, realizada visualmente em torno de dez dias após a inoculação, tendo-se considerada apenas resistência completa e suscetibilidade.

\section{Resultados e Discussão}

As 48 famílias $F_{2: 5}$ passaram por inoculação, inicialmente, da raça 2047 de C. lindemuthianum, para verificar a presença do alelo $\mathrm{Co}-4^{2}$; foram identificadas 15 famílias resistentes, 27 segregantes e seis suscetíveis (Tabela 1), que confirmaram herança monogênica $\left(\chi^{2}=4,12, \mathrm{P}=0,13\right)$.

Nas 15 famílias homozigóticas para o $\mathrm{Co}-4^{2}$, não foi possível identificar também a presença dos alelos Co-5 e Co-7, cujos efeitos foram encobertos pelo amplo espectro de resistência do $\mathrm{Co}-4^{2}$. Uma alternativa para se identificar a presença dos alelos Co-5 e Co-7 seria a utilização de marcadores moleculares. Entretanto, isso não foi possível, porque o alelo $\mathrm{Co}-7$ ainda não foi marcado, e apesar de o alelo Co-5 já ter sido identificado com SCAR SAB03, este marcador não foi eficiente para se praticar a seleção assistida por se encontrar a $12,98 \mathrm{cM}$ desse alelo (Vallejo \& Kelly, 2001) e, provavelmente, já estavam separados na linhagem genitora H91. Couto (2005) utilizou o SCAR desse marcador e verificou baixa eficiência na seleção de genótipos portadores do Co-5, provavelmente pela alta freqüência de recombinação entre eles.

As inoculações das raças 73 e 1545 nas famílias foram feitas independentemente do resultado da inoculação da raça 2047. As 15 famílias portadoras do $\mathrm{Co}-4^{2}$ mostraram-se resistentes às duas raças. Portanto, ficaram definidas as constituições genéticas da maioria das 48 famílias selecionadas (Tabela 1), das quais 15 portadoras do $\mathrm{Co}-4^{2}$, que podem ou não

Tabela 1. Número de famílias identificadas em cada combinação de alelos.

\begin{tabular}{|c|c|}
\hline Combinação dos alelos & Número de famílias \\
\hline $\mathrm{Co}-4^{2}, \mathrm{Co}-5 ?, \mathrm{Co}-7 ?$ & 15 \\
\hline $\mathrm{Co}-4^{2} *, \mathrm{Co}-5^{*}, \mathrm{Co}-7^{*}$ & 13 \\
\hline $\mathrm{Co}-4^{2 *}, \mathrm{Co}-5, \mathrm{Co}-7^{*}$ & 4 \\
\hline $\mathrm{Co}-4^{2} *, \mathrm{Co}-5^{*}, \mathrm{Co}-7$ & 6 \\
\hline $\mathrm{Co}-4^{2 *}, \mathrm{Co}-5, \mathrm{Co}-7$ & 3 \\
\hline $\mathrm{Co}-4^{2 *}, \mathrm{Co}-5^{*}$ & 1 \\
\hline $\mathrm{Co}-5^{*}, \mathrm{Co}-7^{*}$ & 1 \\
\hline $\mathrm{Co}-5, \mathrm{Co}-7^{*}$ & 1 \\
\hline $\mathrm{Co}-5^{*}, \mathrm{Co}-7$ & 2 \\
\hline Co-5 & 1 \\
\hline Co-5* & 1 \\
\hline Total & 48 \\
\hline
\end{tabular}

*Alelos derivados de famílias segregantes para o loco de interesse. 
ter a pirâmide do $C o-5$ e Co-7. Das 27 famílias segregantes para o $\mathrm{Co}-4^{2}$, quatro possuem o $\mathrm{Co}-5$, seis o $\mathrm{Co}-7$, e três, ambos. As 14 restantes podem não ter os alelos $C o-5$ e $C o-7$, porque foram segregantes para as três raças. Outras combinações também foram identificadas: quatro com $\mathrm{Co}-5$ e $\mathrm{Co}-7$ e duas portadoras apenas do Co-5. As famílias portadoras do $\mathrm{Co}-4^{2}$ são as mais promissoras no processo de melhoramento, pois até o momento, no Brasil, não se têm relatos de que a resistência conferida por esse alelo tenha sido quebrada.

As constituições genéticas das famílias foram identificadas com a inoculação das três raças utilizadas $(1545,73$ e 2047) porque, segundo Young et al. (1998), a 1545 quebra a resistência de genótipos com o alelo Co-5. Além disso, de acordo com Alzate-Marin et al. (2001), a raça 73 vence a resistência conferida pelo alelo $\mathrm{Co}-7$, e não vence a conferida pelo $\mathrm{Co}-5$. A raça 2047 somente não vence a resistência conferida pelo alelo $\mathrm{Co}^{-} 4^{2}$.

Os trabalhos de levantamento das raças de C. lindemuthianum mais freqüentemente distribuídas no Brasil indicam que o alelo $\mathrm{Co}-7$ é pouco eficiente para a proteção de cultivares e tem importância menor no processo de melhoramento (Alzate-Marin \& Sartorato, 2004; Ishikawa et al., 2005; Thomazella et al., 2005). De acordo com esses trabalhos, as raças mais freqüentes são também aquelas portadoras do alelo de virulência $2^{6}$, tais como $87,81,73$ e 65 , que quebram a resistência conferida pelo alelo $\mathrm{Co}-7$. Entretanto, as famílias possuidoras deste alelo podem ser isoladas para verificação de seu espectro de resistência às raças disponíveis e, também, para realização de estudos de alelismo.
Em relação às avaliações realizadas em campo, as famílias foram geneticamente diferentes em todos os caracteres, à exceção do porte, na safra de inverno 2004 , e da produção de grãos, na seca de 2005. Serão considerados, a seguir, apenas os resultados das análises conjuntas que envolveram as 48 famílias selecionadas e que foram comuns em todos os experimentos.

As famílias foram geneticamente diferentes $(\mathrm{p} \leq 0,01)$ na média dos locais (Tabela 2$)$. As notas de severidade de mancha-angular variaram de 1,39 a 6,9 , e foi possível selecionar famílias com elevado nível de resistência. Também foi verificada a interação famílias $\mathrm{x}$ ambientes e isso indica um comportamento não coincidente das famílias nos dois ambientes. Esse comportamento pode significar ocorrência de raças diferentes nos dois locais (Lavras e Lambari), já que P. griseola possui ampla variabilidade patogênica (Oliveira et al., 2004). A suscetibilidade da cultivar BRSMG Talismã, utilizada no experimento como testemunha, ficou evidenciada por sua nota 6,05 (Tabela 2).

Quanto ao tipo de grão, também ocorreram diferenças genéticas significativas entre as famílias $(\mathrm{p} \leq 0,01)$, o que indica a possibilidade de selecionar as superiores (Tabela 2). Nesse caso, não se observou a interação de famílias x ambientes, embora o efeito de ambientes tenha sido significativo, o que indica que o ambiente influencia o tipo de grão, mas não altera a classificação das famílias nas diferentes safras. As famílias apresentaram médias de 2,5 a 3,42, e nenhuma delas suplantou a cultivar testemunha BRSMG Talismã, que tem como um de seus melhores atributos o grão tipo 'Carioca'. A estimativa de

Tabela 2. Resumo das análises de variância conjunta para mancha-angular (notas 1-9), tipo de grão (notas 1-5), porte (notas 1-5) e produção $\left(\mathrm{kg} \mathrm{ha}^{-1}\right)$, e estimativas de herdabilidade, considerando-se as safras de inverno de 2004 e das águas de 2004/2005, em Lavras, e da seca de 2005, em Lavras e Lambari.

\begin{tabular}{lcccc}
\hline Fonte de variação & \multicolumn{4}{c}{ Quadrados médios (graus de liberdade) } \\
\cline { 2 - 5 } & Mancha-angular & Tipo de grão & Porte & Produção \\
\hline Famílias (F) & $7,6570^{* *}(48)$ & $0,1947^{* *}(48)$ & $0,1993^{* *}(48)$ & $345.719,4^{* *}(48)$ \\
Ambientes (A) & $0,1667^{\text {ns }}(1)$ & $5,3903^{* *}(2)$ & $1,9425^{* *}(3)$ & $29.488 .424,3^{* *}(3)$ \\
Interação (AxF) & $1,2483^{* *}(48)$ & $0,0573^{\text {ns }}(94)$ & $0,0885^{* *}(141)$ & $189.655,2^{* *}(141)$ \\
Erro-médio & $0,7466(156)$ & $0,0615(335)$ & $0,0552(413)$ & $131.408,6(413)$ \\
\hline Média & 4,85 & 2,96 & 2,59 & $2.669,50$ \\
Média da testemunha & 6,05 & 2,13 & 3,17 & $2.139,95$ \\
DMS (1\%) & 1,1723 & 0,8999 & 0,94555 & $1.384,04$ \\
CV (\%) & 17,81 & 8,06 & 11,47 & 16,31 \\
$\mathrm{~h}^{2}(\%)$ & $90,4[84,1 \mathrm{a} 93,7]^{(1)}$ & $68,4[49,4$ a 78,8$]$ & $72,3[55,8$ a 81,3$]$ & $61,99[39,3$ a 74,4$]$ \\
\hline
\end{tabular}

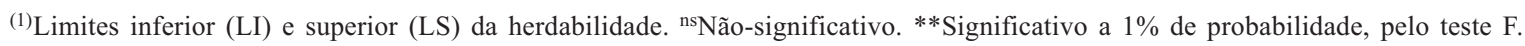


herdabilidade de $68,41 \%$ também sugere a possibilidade de seleção de famílias geneticamente superiores, pelo menos iguais à testemunha, que é considerada de excelente tipo de grão.

Em relação ao porte, também ocorreram diferenças genéticas significativas $(p \leq 0,01)$ entre as famílias, o que indica possibilidade de seleção daquelas com porte mais ereto (Tabela 2). A interação famílias x ambientes foi significativa e mostrou que algumas famílias, que apresentaram porte ereto num dado ambiente, foram decumbentes em outro. A significância dos efeitos de ambientes e da interação ocorreu em razão do grande efeito ambiental que os diferentes ambientes exercem sobre o porte, o que mostra a importância de se avaliar o porte em um maior número de ambientes possível. Na safra das águas, por exemplo, as altas temperaturas e o excesso de chuvas propiciaram o maior crescimento vegetativo das plantas, em conseqüência as plantas tenderam a ficar mais prostradas. A média do porte foi menor do que o porte da testemunha, o que indica que para este caráter a possibilidade de sucesso com a seleção é maior. A estimativa de herdabilidade foi alta, sobretudo pelo fato de o caráter ser muito influenciado pelo ambiente. Couto (2005) encontrou valor superior, mas é importante reforçar que a herdabilidade é mutável e varia com a população e a condição ambiental a que o material genético é submetido.

A produtividade de grãos das 48 famílias também foi geneticamente diferente $(p \leq 0,01)$, tendo possibilitado a seleção de famílias mais produtivas (Tabela 2). O efeito da interação famílias x safras também foi significativo e mostrou comportamento não coincidente das famílias nos diferentes ambientes, certamente em razão da enorme sensibilidade do caráter ao ambiente (Ramalho et al., 1993; Pereira et al., 2004). Entretanto, a ampla diversidade das famílias é expressa pela herdabilidade de $61,99 \%$, superior à observada por Pereira et al. (2004), e permite antever sucesso com a seleção. A produção média de grãos das famílias foi semelhante à da testemunha BRSMG Talismã. Entretanto vale lembrar que as famílias são segregantes e há a possibilidade de seleção de linhagens dentro dessas famílias com produtividade de grãos superior.

Considerando-se a seleção direta das cinco melhores famílias, com base nas médias ajustadas das análises conjuntas para reação à mancha-angular, tipo de grão, porte e produção de grãos, observou-se ganhos para todos os caracteres, com destaque para reação à mancha-angular, em que o ganho foi expressivo (Tabela 3). Entretanto, a seleção com base em apenas uma característica tem-se mostrado inadequada. Assim, a seleção simultânea de um conjunto de caracteres tende a aumentar a chance de êxito de um programa de melhoramento (Cruz \& Carneiro, 2003).

Desse modo, foi considerada a seleção das quatro famílias superiores, tendo-se adotado como critérios: a reação à mancha-angular menor do que 3,8; tipo de grão menor do que 3; porte menor do que 2,8; e produção de grãos maior do que a média das famílias. Em conseqüência, em vez de se obter um ganho de $44,4 \%$ na resistência à mancha-angular, obteve-se $35,6 \%$, que foi, ainda, bastante expressivo. A mesma tendência pode ser observada para todas as outras características e, ainda, de forma mais drástica para porte, em que a média das famílias selecionadas foi praticamente igual à média de todas as famílias (Tabela 3). As quatro famílias selecionadas foram 20, 24, 31 e 33 (Tabela 4). Apesar de não ter sido considerada a resistência à antracnose, nesse procedimento de seleção, todas as quatro famílias são portadoras do alelo $\mathrm{Co}-4^{2}$, duas das quais homozigóticas, que podem possuir também os alelos Co-5 e Co-7, e duas segregantes para o $\mathrm{Co}_{-} 4^{2}$ e portadoras do alelo Co-5. Porém, destaca-se entre elas, a família 20, que possui o melhor tipo de grão da população, porte ereto, produção acima da média, resistência à mancha-angular, e também, o alelo $\mathrm{Co}-4^{2}$ em homozigose, que confere resistência a todas as raças de antracnose já identificadas no Brasil.

A causa para a resposta indireta à seleção, quando mais de um caráter é considerado, é a correlação entre eles. Com base nisso, foram estimadas as correlações fenotípicas entre os caracteres avaliados.

Tabela 3. Estimativas de ganho esperado com a seleção (GS).

\begin{tabular}{lcccc}
\hline Estimativa Mancha-angular & Tipo de grão & Porte & Produção \\
\hline $\mathrm{GS}^{(1)}$ & $-2,15$ & $-0,273$ & $-0,284$ & 323,3 \\
& $(44,4 \%)$ & $(9,21 \%)$ & $(10,9 \%)$ & $(12,11 \%)$ \\
$\mathrm{GS}^{(2)}$ & $-1,72$ & $-0,150$ & $-0,043$ & 170,78 \\
& $(35,56 \%)$ & $(5,06 \%)$ & $(1,68 \%)$ & $(6,4 \%)$ \\
\hline
\end{tabular}

${ }^{(1)}$ Considerando-se as cinco melhores famílias para cada caráter em separado (seleção direta). ${ }^{(2)}$ Considerando-se como critérios para seleção de quatro famílias superiores com reação à mancha-angular $<3,8$; tipo de grão $<3,0$; porte $<2,8$ e produção $>$ média $\left(\mathrm{kg} \mathrm{ha}^{-1}\right)$. 
De acordo com a Tabela 5, nota-se que houve correlação baixa e negativa entre tipo de grão e produção, e correlação baixa e positiva entre porte e produção, ou seja, as famílias mais produtivas são aquelas que possuem um tipo de grão de maior aceitação e um porte inferior. Para as demais estimativas, as correlações

Tabela 4. Médias ajustadas de reação à mancha-angular, tipo de grão, porte, produção de grãos e alelos de resistência à antracnose, presentes nas 48 famílias utilizadas nas análises conjuntas ${ }^{(1)}$.

\begin{tabular}{|c|c|c|c|c|c|}
\hline Família & $\begin{array}{c}\text { Mancha-angular } \\
(1-9)\end{array}$ & $\begin{array}{l}\text { Tipo de grão } \\
(1-5)\end{array}$ & $\begin{array}{l}\text { Porte } \\
(1-5)\end{array}$ & Produção $\left(\mathrm{kg} \mathrm{ha}^{-1}\right)$ & Alelo de resistência \\
\hline 1 & $5,38 \mathrm{a}$ & $3,29 \mathrm{a}$ & $2,91 \mathrm{a}$ & $2.707,8 \mathrm{a}$ & $\mathrm{Co}-4^{2 *}, \mathrm{Co}-5, \mathrm{Co}-7^{*} ?$ \\
\hline 2 & $5,64 \mathrm{a}$ & $2,94 \mathrm{a}$ & $2,73 a$ & $2.659,4 \mathrm{~b}$ & $\mathrm{Co}-4^{2} *, \mathrm{Co}-5^{*} ?, \mathrm{Co}-7^{*} ?$ \\
\hline 3 & $5,66 \mathrm{a}$ & $2,74 b$ & $2,74 a$ & $3.018,0 \mathrm{a}$ & Co- $5^{*}$ \\
\hline 4 & $5,57 \mathrm{a}$ & $3,12 \mathrm{a}$ & $2,70 \mathrm{a}$ & $2.552,0 \mathrm{~b}$ & $\mathrm{Co}-4^{2} *, \mathrm{Co}-5^{*} ?, \mathrm{Co}-7^{*} ?$ \\
\hline 5 & $4,82 \mathrm{a}$ & $3,25 \mathrm{a}$ & $2,79 a$ & $2.912,6 \mathrm{a}$ & $\mathrm{Co}-4^{2} *, \mathrm{Co}-5 * ?, \mathrm{Co}-7^{*} ?$ \\
\hline 6 & $4,66 a$ & $2,60 \mathrm{~b}$ & $2,76 \mathrm{a}$ & $2.374,9 b$ & $\mathrm{Co}-4^{2} *, \mathrm{Co}-5, \mathrm{Co}-7 * ?$ \\
\hline 7 & $5,40 \mathrm{a}$ & $3,32 \mathrm{a}$ & $2,36 b$ & $2.420,2 \mathrm{~b}$ & $\mathrm{Co}-4^{2} *, \mathrm{Co}-5^{*} ?, \mathrm{Co}-7^{*} ?$ \\
\hline 8 & $5,01 \mathrm{a}$ & $2,57 b$ & $2,74 a$ & $3.246,3 a$ & $\mathrm{Co}^{-} 5^{*}, \mathrm{Co}-7^{*}$ \\
\hline 9 & $4,95 \mathrm{a}$ & $2,70 \mathrm{~b}$ & $2,23 b$ & $2.824,3 \mathrm{a}$ & $\mathrm{Co}-4^{2}, \mathrm{Co}-5 ?, \mathrm{Co}-7 ?$ \\
\hline 10 & $6,52 \mathrm{a}$ & $3,43 a$ & $2,91 \mathrm{a}$ & $2.637,2 \mathrm{~b}$ & $\mathrm{Co}-5$ \\
\hline 11 & $5,28 \mathrm{a}$ & $3,18 \mathrm{a}$ & $3,18 \mathrm{a}$ & $3.469,9 \mathrm{a}$ & Co $-4^{2 *}$, Co $-5 * ?$ \\
\hline 12 & $5,27 \mathrm{a}$ & $2,66 b$ & $2,52 \mathrm{~b}$ & $2.791,9 \mathrm{a}$ & $\mathrm{Co}-4^{2 *}, \mathrm{Co}-5 * ?, \mathrm{Co}-7 * ?$ \\
\hline 13 & $4,86 a$ & $3,05 \mathrm{a}$ & $2,50 \mathrm{~b}$ & $2.875,7 \mathrm{a}$ & $\mathrm{Co}-4^{2 *}, \mathrm{Co}-5, \mathrm{Co}-7 * ?$ \\
\hline 14 & $4,64 \mathrm{a}$ & $3,20 \mathrm{a}$ & $2,63 \mathrm{a}$ & $2.413,8 b$ & $\mathrm{Co}-4^{2}, \mathrm{Co}-5 ?, \mathrm{Co}-7 ?$ \\
\hline 15 & $4,21 \mathrm{~b}$ & $2,83 \mathrm{~b}$ & $2,31 \mathrm{~b}$ & $2.414,3 b$ & $\mathrm{Co}-4^{2 *}, \mathrm{Co}-5^{*} ?, \mathrm{Co}-7^{*}$ ? \\
\hline 16 & $4,87 \mathrm{a}$ & $2,52 b$ & $2,77 \mathrm{a}$ & $2.450,9 \mathrm{~b}$ & $\mathrm{Co}-4^{2 *}, \mathrm{Co}-5^{*} ?, \mathrm{Co}-7$ \\
\hline 17 & $4,79 a$ & $2,60 \mathrm{~b}$ & $2,70 \mathrm{a}$ & $3.055,1 \mathrm{a}$ & Сo- $4^{2}, \mathrm{Co}-5 ?, \mathrm{Co}-7 ?$ \\
\hline 18 & $3,93 b$ & $3,34 \mathrm{a}$ & $2,59 \mathrm{a}$ & $2.704,2 \mathrm{a}$ & $\mathrm{Co}-4^{2} *, C o-5$, Co- 7 \\
\hline 19 & $5,26 \mathrm{a}$ & $3,02 \mathrm{a}$ & $2,30 \mathrm{~b}$ & $2.534,1 \mathrm{~b}$ & $\mathrm{Co}-4^{2 *}, \mathrm{Co}-5 * ?, \mathrm{Co}-7 * ?$ \\
\hline 20 & $3,27 \mathrm{~b}$ & $2,57 \mathrm{~b}$ & $2,46 b$ & $2.867,3 \mathrm{a}$ & $\mathrm{Co}-4^{2}, \mathrm{Co}-5 ?, \mathrm{Co}-7 ?$ \\
\hline 21 & $2,80 \mathrm{~b}$ & $3,15 \mathrm{a}$ & $2,74 a$ & $2.633,1 \mathrm{~b}$ & $\mathrm{Co}-4^{2}, \mathrm{Co}-5 ?, \mathrm{Co}-7 ?$ \\
\hline 22 & $6,11 \mathrm{a}$ & $3,05 \mathrm{a}$ & $2,76 \mathrm{a}$ & $2.508,3 \mathrm{~b}$ & $\mathrm{Co}-4^{2 *}, \mathrm{Co}-5, \mathrm{Co}-7$ \\
\hline 23 & $5,15 \mathrm{a}$ & $2,73 b$ & $2,46 b$ & $2.796,1 \mathrm{a}$ & $\mathrm{Co}-4^{2}, \mathrm{Co}-5 ?, \mathrm{Co}-7 ?$ \\
\hline 24 & $3,29 b$ & $2,74 b$ & $2,83 \mathrm{a}$ & $3.005,6 \mathrm{a}$ & $\mathrm{Co}-4^{2} * \mathrm{Co}-5, \mathrm{Co}-7$ \\
\hline 25 & $3,27 \mathrm{~b}$ & $2,88 \mathrm{~b}$ & $2,09 \mathrm{~b}$ & $2.441,6 \mathrm{~b}$ & $\mathrm{Co}-4^{2}, \mathrm{Co}-5 ?, \mathrm{Co}-7 ?$ \\
\hline 26 & $5,99 \mathrm{a}$ & $2,96 \mathrm{a}$ & $2,47 b$ & $2.248,6 \mathrm{~b}$ & $\mathrm{Co}-4^{2}, \mathrm{Co}-5 ?, \mathrm{Co}-7 ?$ \\
\hline 27 & $4,51 \mathrm{a}$ & $3,06 \mathrm{a}$ & $2,63 a$ & $2.353,5 b$ & $\mathrm{Co}-4^{2 *}, \mathrm{Co}-5^{*} ?, \mathrm{Co}-7$ \\
\hline 28 & $5,28 \mathrm{a}$ & $2,63 b$ & $2,81 \mathrm{a}$ & $2.691,7 \mathrm{a}$ & $\mathrm{Co}-4^{2 *}, \mathrm{Co}-5^{*} ?, \mathrm{Co}-7^{*}$ ? \\
\hline 29 & $5,98 \mathrm{a}$ & $2,85 \mathrm{~b}$ & $2,51 \mathrm{~b}$ & $2.547,7 \mathrm{~b}$ & $\mathrm{Co}-4^{2}, \mathrm{Co}-5 ?, \mathrm{Co}-7 ?$ \\
\hline 30 & $2,94 b$ & $3,00 \mathrm{a}$ & $2,18 \mathrm{~b}$ & $2.495,6 \mathrm{~b}$ & $\mathrm{Co}-4^{2 *}, \mathrm{Co}-5^{*} ?, \mathrm{Co}-7$ \\
\hline 31 & $3,79 b$ & $2,67 \mathrm{~b}$ & $2,20 \mathrm{~b}$ & $3.165,6 \mathrm{a}$ & $\mathrm{Co}-4^{2 *}, \mathrm{Co}-5$ e $\mathrm{Co}-7 * ?$ \\
\hline 32 & $5,00 \mathrm{a}$ & $2,59 b$ & $2,83 a$ & $2.862,9 \mathrm{a}$ & $\mathrm{Co}-4^{2} *, \mathrm{Co}-5^{*} ?, \mathrm{Co}-7^{*} ?$ \\
\hline 33 & $1,39 \mathrm{c}$ & $3,04 \mathrm{a}$ & $2,64 \mathrm{a}$ & $2.741,5 \mathrm{a}$ & $\mathrm{Co}-4^{2}, \mathrm{Co}-5 ?, \mathrm{Co}-7 ?$ \\
\hline 34 & $4,13 b$ & $3,35 \mathrm{a}$ & $2,68 \mathrm{a}$ & $2.238,0 \mathrm{~b}$ & $\mathrm{Co}-4^{2}, \mathrm{Co}-5 ?, \mathrm{Co}-7 ?$ \\
\hline 35 & $4,57 \mathrm{a}$ & $3,33 a$ & $2,71 \mathrm{a}$ & $2.931,4 \mathrm{a}$ & $\mathrm{Co}_{\mathrm{S}} \mathrm{F}^{*}, \mathrm{Co}-\mathrm{7}^{*}$ \\
\hline 36 & $5,03 \mathrm{a}$ & $2,83 b$ & $2,47 b$ & $2.883,3 \mathrm{a}$ & $\mathrm{Co}-4^{2 *}, \mathrm{Co}-5^{*} ?, \mathrm{Co}-7^{*} ?$ \\
\hline 37 & $5,90 \mathrm{a}$ & $3,03 \mathrm{a}$ & $2,50 \mathrm{~b}$ & $2.805,0 \mathrm{a}$ & $\mathrm{Co}-5^{*}, \mathrm{Co}-7$ \\
\hline 38 & $4,17 b$ & $2,89 \mathrm{~b}$ & $2,38 \mathrm{~b}$ & $2.433,8 \mathrm{~b}$ & $\mathrm{Co}-4^{2} * \mathrm{Co}-5^{*} ? \mathrm{Co}-7$ \\
\hline 39 & $4,59 a$ & $3,18 \mathrm{a}$ & $2,66 \mathrm{a}$ & $2.915,3 \mathrm{a}$ & $\mathrm{Co}-4^{2 *}, \mathrm{Co}-5, \mathrm{Co}-7 * ?$ \\
\hline 40 & $6,20 \mathrm{a}$ & $2,98 \mathrm{a}$ & $2,41 b$ & $2636,5 b$ & $\mathrm{Co}-4^{2} *, \mathrm{Co}-5^{*} ?, \mathrm{Co}-7^{*} ?$ \\
\hline 41 & $5,29 a$ & $3,23 \mathrm{a}$ & $2,89 \mathrm{a}$ & $2.322,5 b$ & $\mathrm{Co}-4^{2 *}, \mathrm{Co}-5^{*} ?, \mathrm{Co}-7^{*} ?$ \\
\hline 42 & $1,95 \mathrm{c}$ & $3,16 \mathrm{a}$ & $2,55 \mathrm{~b}$ & $2.359,9 \mathrm{~b}$ & $\mathrm{Co}-4^{2}, \mathrm{Co}-5 ?, \mathrm{Co}-7 ?$ \\
\hline 43 & $5,18 \mathrm{a}$ & $3,19 a$ & $2,44 b$ & $2.297,9 \mathrm{~b}$ & $\mathrm{Co}-4^{2}, \mathrm{Co}-5 ?, \mathrm{Co}-7 ?$ \\
\hline 44 & $5,56 a$ & $3,28 \mathrm{a}$ & $2,63 \mathrm{a}$ & $2.471,2 \mathrm{~b}$ & $\mathrm{Co}-4^{2 *}, \mathrm{Co}-5^{*} ?, \mathrm{Co}-7$ \\
\hline 45 & $5,91 \mathrm{a}$ & $2,75 b$ & $2,84 \mathrm{a}$ & $2.773,3 \mathrm{a}$ & $\mathrm{Co}^{\circ} 5^{*}, \mathrm{Co}-7$ \\
\hline 46 & $5,91 \mathrm{a}$ & $3,01 \mathrm{a}$ & $2,51 \mathrm{~b}$ & $2.887,6 \mathrm{a}$ & $\mathrm{Co}-4^{2}, \mathrm{Co}-5 ?, \mathrm{Co}-7 ?$ \\
\hline 47 & $6,90 a$ & $2,84 b$ & $2,54 b$ & $2.826,7 \mathrm{a}$ & $\mathrm{Co}-4^{2 *}, \mathrm{Co}-5^{*} ?, \mathrm{Co}-7^{*} ?$ \\
\hline 48 & $5,04 \mathrm{a}$ & $3,15 \mathrm{a}$ & $2,30 \mathrm{~b}$ & $1.932,1 \mathrm{~b}$ & $\mathrm{Co}-4^{2}, \mathrm{Co}-5 ?, \mathrm{Co}-7 ?$ \\
\hline
\end{tabular}

${ }^{(1)}$ Médias seguidas de letras iguais são semelhantes pelo teste de Scott-Knott a $5 \%$ de probalidade. *Alelos derivados de famílias segregantes para o loco de interesse; ? indica possibilidade das famílias terem ou não os alelos de resistência. 
Tabela 5. Correlações fenotípicas entre os caracteres avaliados.

\begin{tabular}{lccc}
\hline Carácter & Tipo de grão & Porte & Produção \\
\hline Mancha-angular & $0,017^{\text {ns }}$ & $0,206^{\text {ns }}$ & $0,043^{\text {ns }}$ \\
Tipo de grão & & $0,136^{\text {ns }}$ & $-0,314^{*}$ \\
Porte & & & $0,315^{*}$ \\
\hline
\end{tabular}

nsNão-significativo. ${ }^{*}$ Significativo a $5 \%$ de probabilidade, pelo teste t.

foram não significativas, o que indica a possibilidade de seleção de famílias que sejam superiores em todos os caracteres avaliados.

\section{Conclusões}

1. Existe ampla variabilidade das famílias de feijão, principalmente quanto à reação à mancha-angular.

2. As famílias com elevada resistência à manchaangular são portadoras do alelo de resistência proveniente da cultivar andina Jalo EEP 558.

3. Das quatro famílias com tipo de grão semelhante ao carioca, porte ereto, produtividade superior, resistência à mancha-angular e portadoras do alelo de resistência à antracnose $\mathrm{Co}_{-} 4^{2}$, pelo menos uma possui também os alelos $\mathrm{Co}-5$ e $\mathrm{Co}-7$.

\section{Agradecimentos}

À Capes, pela concessão da bolsa de estudos; à Fapemig, pelo apoio financeiro.

\section{Referências}

ALZATE-MARIN, A.L.; MENARIM, H.; BAÍA, G.S.; PAULA JUNIOR, J.T.; SOUZA, K.A. de; COSTA, M.R. da; BARROS, E.G. de; MOREIRA, M.A. Inheritance of anthracnose resistance in the common bean differential cultivar G 2333 and identification of a new molecular marker linked to the $\mathrm{Co}-4^{2}$ gene. Journal of Phytopathology, v.149, p.259-264, 2001.

ALZATE-MARIN, A.L.; SARTORATO, A. Analysis of the pathogenic variability of Colletotrichum lindemuthianum in Brazil. Annual Report of the Bean Improvement Cooperative, v.47, p.241-242, 2004.

BRUZI, A.T.; SILVA, F.B.; RAMALHO, M.A.P.; SANTOS, J.B. dos. Performance of common bean families from crossing of Andean and Mesoamerican lines. Annual Report of the Bean Improvement Cooperative, v.47, p.299-300, 2004.

COLliCCHIO, E.; RAMALHO, M.A.P.; ABREU, A.F.B. Associação entre o porte da planta do feijoeiro e o tamanho dos grãos. Pesquisa Agropecuária Brasileira, v.32, p.297-304, 1997.
COUTO, M.A. Seleção de linhagens de feijão tipo carioca com resistência à antracnose e à mancha angular. 2005. 74p. Dissertação (Mestrado) - Universidade Federal de Lavras, Lavras.

CRUZ, C.D.; CARNEIRO, P.C.S. Modelos biométricos aplicados ao melhoramento genético. Viçosa: UFV, 2003. v.2. 585p.

INDICADORES DA AGROPECUÁRIA. Brasília: Conab, v.14, 2005. 60p.

ISHIKAWA, F.H.; SILVA, K.J.D.; SOUZA, E.A.; DAVIDE, L.M.C.; FREIRE, C.N.S. Levantamento de raças de Colletotrichum lindemuthianum de regiões produtoras de feijão. In: CONGRESSO NACIONAL DE PESQUISA DE FEIJÃO, 8., 2005, Goiânia. Anais. Santo Antônio de Goiás: Embrapa Arroz e Feijão, 2005. p.501-504. (Embrapa Arroz e Feijão. Documentos, 182).

KELLY, J.D.; MIKLAS, P.N. The role of RAPD markers in breeding for disease resistance in common bean. Molecular Breeding, v.4, p.1-11, 1998.

KNAPP, S.J.; STROUP, W.W.; ROSS, W.M. Exact confidence intervals for heritability on a progeny mean basis. Crop Science, v.25, p.192-194, 1985.

MARQUES JUNIOR, O.G. Eficiência de experimento com a cultura do feijão. 1997. 80p. Tese (Doutorado) - Universidade Federal de Lavras, Lavras.

OLIVEIRA, E.J. de; ALZATE-MARIN, A.L.; BORÉM, A.; MELO, C.L.P.; BARROS, E.G.; MOREIRA, M.A. Reação de cultivares de feijoeiro comum a quatro raças de Phaeoisariopsis griseola. Fitopatologia Brasileira, v.29, p.220-223, 2004.

PELOSO, M.J. Antracnose do feijoeiro no Estado de Minas GeraisBrasil. In: PASTOR-CORRALES, M.A. (Ed.). La antracnosis del frijol común, Phaseolus vulgaris, en América Latina. Cali: CIAT, 1992. (Doc. de trabajo, 113).

PEREIRA, H.S.; SANTOS, J.B. dos; ABREU, A. de F.B. Linhagens de feijoeiro com resistência à antracnose selecionadas quanto a características agronômicas desejáveis. Pesquisa Agropecuária Brasileira, v.39, p.209-215, 2004.

RAMALHO, M.A.P.; SANTOS, J.B. dos; ZIMMERMANN, J.O. Genética quantitativa em plantas autógamas. Goiânia: UFG, 1993. 271p.

RAVA, C.; PURCHIO, A.; SARTORATO, A. Caracterização de patótipos de Colletotrichum lindemuthianum que ocorrem em algumas regiões produtoras de feijoeiro comum. Fitopatologia Brasileira, v.19, p.167-172, 1994.

SARTORATO, A. Resistance of Andean and Mesoamerican common bean genotypes to Phaeoisariopsis griseola. Annual Report of the Bean Improvement Cooperative, v.48, p.88-89, 2005.

THOMAZELLA, C.; VIDIGAL, M.C.G.; VIDIGAL-FILHO, P.S.; ELIAS, H.T.; GONELA, A.; POLETINE, J.P. Characterization of Colletotrichum lindemuthianum isolates from Santa Catarina State. 
In: CONGRESSO NACIONAL DE PESQUISA DE FEIJÃO, 8., 2005, Goiânia. Anais. Santo Antônio de Goiás: Embrapa Arroz e Feijão, 2005. p.363-366. (Embrapa Arroz e Feijão. Documentos, 182).

VALLEJO, V.; KELLY, J.D. Development of a SCAR marker linked to $\mathrm{Co}-5$ locus in common bean. Annual Report of the Bean Improvement Cooperative, v.44, p.121-122, 2001.
VAN SCHOONHOVEN, A.; PASTOR-CORRALES, M.A. (Comps.). Standard system for evaluation of bean germplasm. Cali: CIAT, 1987. 54p.

YOUNG, R.A.; MELOTTO, M.; NODARI, R.O.; KELLY, J.D. Marker-assisted dissection of the oligogenic anthracnose resistance in the common bean cultivar, 'G2333'. Theoretical and Applied Genetics, v.96, p.87-94, 1998.

$\overline{\text { Recebido em 1o de novembro de } 2005 \text { e aprovado em } 19 \text { de junho de } 2006}$ 